\title{
CARACTERIZAÇÃo DE ADUBOS ORGÂNICOS POR ESPECTROSCOPIA DE RESSONÂNCIA PARAMAGNÉTICA ELETRÔNICA ${ }^{(1)}$
}

\author{
Marcelo Luiz Simões ${ }^{(2)}$, Wilson Tadeu Lopes da Silva ${ }^{(3)}$, \\ Sergio da Costa Saab ${ }^{(4)}$, Larissa Macedo dos Santos $^{(5)} \&$ \\ Ladislau Martin-Neto ${ }^{(6)}$
}

\begin{abstract}
RESUMO
O aumento do interesse na produção dos alimentos denominados orgânicos fez crescer a demanda por insumos com características adequadas para atender a esse sistema de produção. Contudo, a qualidade dos denominados adubos orgânicos disponíveis para comercialização é, em alguns casos, questionável devido à falta de uma metodologia de análise que avalie o produto. Neste trabalho é apresentada uma proposta de metodologia baseada na avaliação do grau de humificação inferido por meio da quantificação dos radicais livres orgânicos (RLO), presentes na matéria orgânica, e que podem ser detectados por meio da espectroscopia de ressonância paramagnética eletrônica (RPE). Foram utilizadas nove amostras de adubos orgânicos de diferentes procedências e, como possíveis fontes de adulteração, dois solos e carvão vegetal. Os resultados mostraram significativa variação na concentração de RLO entre os diferentes adubos orgânicos analisados (de $0,10 \times 10^{18}$ spins $\mathrm{g}^{-1}$ a $1,84 \times 1^{18}$ spins $\left.\mathrm{g}^{-1}\right)$. A análise de componentes principais (PCA) confirmou a significância estatística entre as amostras com relação a esse parâmetro, podendo este ser utilizado para diferenciar os adubos orgânicos. Possíveis adulterações, provindas da adição de solos e carvão vegetal, foram detectadas pela observação de alterações nos espectros de RPE, como concentração
\end{abstract}

\footnotetext{
(1) Recebido para publicação em maio de 2005 e aprovado em janeiro de 2007.

(2) Assistente A da Embrapa Instrumentação Agropecuária. Caixa Postal 741, CEP 13560-970 São Carlos (SP). E-mail: marcelo@cnpdia.embrapa.br

(3) Analista A da Embrapa Instrumentação Agropecuária. Caixa Postal 741, CEP 13560-970 São Carlos (SP). E-mail: wilson@cnpdia.embrapa.br

(4) Professor Adjunto do Departamento de Física da Universidade Estadual de Ponta Grossa - UEPG. Av. Carlos Cavalcanti 4748, CEP 84030-900 Ponta Grossa (PR). E-mail: scsaab@uepg.br

(5) Doutorando do Programa de Pós-Graduação do Instituto de Química de São Carlos. Caixa Postal 369, CEP $13560-970$ São Carlos (SP). E-mail: larissa@cnpdia.embrapa.br

(6) Pesquisador da Embrapa Instrumentação Agropecuária. Caixa Postal 741, CEP 13560-970 São Carlos (SP). E-mail: martin@cnpdia.embrapa.br
} 
de spins, largura de linha do sinal, fator $\mathrm{g}$, forma de linha e surgimento de novos sinais. O limite de detecção de impurezas variou em torno de 5 e $10 \%$, dependendo do tipo de impureza adicionada e do parâmetro espectral analisado.

Termos de indexação: adubo orgânico, agricultura orgânica, radicais livres orgânicos, humificação, RPE.

\title{
SUMMARY: CHARACTERIZATION OF ORGANIC FERTILIZERS BY ELECTRON PARAMAGNETIC RESONANCE
}

\begin{abstract}
The growing interest in the production of organic foods has intensified the demand for fertilizers with suitable characteristics to supply this production system. However, the quality of commercial organic fertilizers is, in some cases, doubtful due to the lack of analytical methodology to evaluate the products. Our study proposes a methodology based on the evaluation of the humification degree obtained by quantification of organic free radicals (OFR), which are naturally present in organic matter and can be detected by electronic paramagnetic resonance (EPR). Nine samples of organic fertilizers of different origins were analyzed and two soils and vegetal coal were used as possible sources of adulteration. The results showed substantial variations in the OFR concentration in the samples (from $0.10 \times 10^{18}$ to $1.84 \times 10^{18}$ spins $g^{-1}$ ). The Principal Component Analysis (PCA) confirmed the statistically significant difference among samples for this parameter. This indicates that OFR concentration can be used to differentiate organic fertilizers. Analyzing spectral alterations in the EPR signals such as spin concentration, line width, $g$ value, line shape and detection of new signals, it was also possible to detect adulterations in the samples caused by soil and vegetal coal additions. The detection limits of the adulterations were around $5 \%$ and $10 \%$, depending on the type of impurity and EPR parameter under study.
\end{abstract}

Index terms: organic fertilizers, organic agriculture, organic free radicals, humification, $E P R$.

\section{INTRODUÇÃO}

O interesse pela produção de adubos orgânicos no Brasil tem aumentado significativamente nos últimos anos, devido principalmente à busca de alternativas de manejo do solo com enfoque orgânico e com aspectos distintos do sistema convencional de uso intensivo de fertilizantes químicos. Esse interesse está estreitamente relacionado à expansão do mercado de produtos orgânicos, que, segundo a Associação de Agricultura Orgânica (AAO), já havia faturado US\$ 150 milhões em 1999. De acordo com a AAO, produtos orgânicos são aqueles em que o sistema de produção exclui o uso de fertilizantes sintéticos de alta solubilidade e pesticidas. Nesse sentido, torna-se importante caracterizar os adubos orgânicos tanto com relação ao material de origem, que pode conter metais pesados (Tomati et al., 2002), quanto para definir em quais propriedades (física, química ou biológica) irão atuar os inúmeros produtos já disponíveis no mercado. No entanto, constata-se que existe demanda por uma regulamentação mínima que estabeleça um padrão de qualidade dos materiais produzidos. Por exemplo, a revista Globo Rural de outubro de 1996, páginas 29 a 33, relatou que produtores de húmus de minhoca estão encontrando competidores que claramente adulteram seus produtos com o intuito de aumentar a sua quantidade produzida e comercializada.

A adulteração inclui a mistura de solo, o qual não se constitui um condicionador físico e químico, descaracterizando a ação potencial do adubo orgânico. Outra fonte de adulteração a ser considerada é a oriunda da adição de carvão vegetal. Isso se deve ao fato de que, apesar de trabalhos terem mostrado que os subprodutos dessa produção (fino de carvão e extrato pirolenhoso) são promissores para utilização na agricultura (Tsuzuki et al., 2000; Miyasaka et al., 2001); Zanetti et al. (2003), utilizando os subprodutos da produção do carvão vegetal na formação de portaenxerto de limoeiro Citrus limonia Osbeck, verificaram diferentes influências no desenvolvimento das plantas. O tipo de influência observada por Zanetti et al. (2003) dependeu da proporção aplicada, porém nenhum efeito benéfico foi observado. Além disso, o carvão vegetal, quando analisado por ressonância paramagnética eletrônica (RPE), também apresenta sinal de radical livre orgânico (RLO), e a concentração desses radicais livres normalmente é alta. Dessa forma, tanto solos como carvão vegetal, se misturados às amostras de adubos orgânicos, podem ocasionar 
alterações na concentração de RLO, gerando valores subestimados ou superestimados, influenciando na avaliação do grau de humificação.

A humificação é definida como a transformação de fontes estruturalmente identificáveis (açúcares, polissacarídeos, proteínas etc.) em compostos húmicos amorfos, e o grau de humificação é um parâmetro importante para determinação da qualidade da matéria orgânica do solo (MOS) (Zech et al., 1997; Bayer et al., 2002) e de resíduos orgânicos (Rovira et al., 2002; Plaza et al., 2005). Em relação à resposta de uma dada cultura, a diferença entre aplicar diretamente resíduos orgânicos estabilizados e nãoestabilizados está na quantidade e época em que os nutrientes mineralizados estarão disponíveis para as plantas, assim como na intensidade com que ativam os microrganismos do solo. No caso de adubos orgânicos que têm como objetivo aumentar a capacidade de troca catiônica (CTC) do solo, o ideal é a aplicação de material já humificado (Plaza et al., 2003; Grigatti et al., 2004). Além disso, se o composto não estiver estabilizado, pode causar efeitos tóxicos para as plantas (Atiyeh et al., 2001).

O grau de humificação da MOS tem sido inferido por meio da determinação da concentração de RLO detectáveis por RPE (Riffaldi \& Schnitzer, 1972; Schnitzer \& Levesque, 1979; Martin-Neto et al., 1998; Jerzykiewicz et al., 1999; Saab \& Martin-Neto, 2003). Tem sido mostrado que a concentração desses radicais aumenta à medida que o processo de humificação avança (Martin-Neto et al., 1991, 1994, 1998; Ceretta et al., 1999; Olk et al., 2000; Dick et al., 2002; Pajaczkowska et al., 2003; Perez et al., 2004). Assim, a informação pode também se tornar um parâmetro de qualificação e distinção para adubos orgânicos.

Com base nessas informações, este trabalho teve como objetivo avaliar a possibilidade de utilização da espectroscopia de RPE para diferenciar adubos orgânicos e detectar adulterações provindas da adição de solos e carvão vegetal.

\section{MATERIAL E MÉTODOS}

Foram utilizadas amostras de nove adubos orgânicos, cujos números, procedências e descrições encontram-se no quadro 1.

Para verificar a sensibilidade da análise na determinação de possíveis adulterações, dois solos (Quadro 2) e carvão vegetal foram adicionados a duas amostras (6 e 7). Nas adições (adubos + solos ou carvão vegetal) obteve-se massa total de aproximadamente 20 g nas seguintes porcentagens de solos ou carvão: $1,5,10$ e $20 \%(\mathrm{~m} / \mathrm{m})$. Após a adição, as amostras foram homogeneizadas mecanicamente, utilizando o equipamento Vribrating Mill, marca Perkin-Elmer, e posteriormente analisadas por RPE.

\section{Quadro 1. Número, procedência e descrição das amostras dos adubos orgânicos}

\begin{tabular}{cll}
\hline Identificação & \multicolumn{1}{c}{ Tipo do produto } & \multicolumn{1}{c}{ Descrição do produto } \\
\hline 1 & Sistema de biodigestão-produto não-comercial & Composto orgânico biodigerido \\
2 & Produto comercial & Esterco bovino \\
3 & Produto comercial importado & Ácidos húmicos e fúlvicos de turfa \\
4 & Produto comercial & Composto orgânico \\
5 & Produto comercial & Vermicomposto \\
6 & Produto não-comercial & Composto orgânico de leonardita \\
7 & Produto comercial & Composto orgânico \\
8 & Produto comercial & Substrato orgânico \\
9 & Produto comercial & Terra vegetal \\
\hline
\end{tabular}

Quadro 2. Características granulométricas, teores de ferro disponível e total e matéria orgânica (MO) dos solos utilizados

\begin{tabular}{|c|c|c|c|c|c|c|c|}
\hline Tipo de solo & Identificação & Argila & Silte & Areia & Fe disponível & Fe total & MO \\
\hline & & $\ldots$ & $\mathrm{g} \mathrm{kg}^{-1}$ & $\underline{-}$ & $\mathrm{mg} \mathrm{kg} \cdot 1$ & $\mathrm{~g} \mathrm{~kg}^{-1}$ & $\mathrm{~g} \mathrm{dm}^{-3}$ \\
\hline Latossolo Vermelho & $\mathrm{S} 1$ & 426 & 61 & 513 & 31 & 98,02 & 22 \\
\hline Latossolo Amarelo & $\mathrm{S} 2$ & 141 & 40 & 819 & 74 & 1,43 & 17 \\
\hline
\end{tabular}


Para obtenção dos espectros de RPE foi utilizado o espectrômetro marca Bruker EMX, operando em banda $\mathrm{X}(9 \mathrm{GHz})$, à temperatura ambiente. Para análise quantitativa, tubos de quartzo foram preenchidos com amostras de adubos orgânicos, anotando-se as respectivas massas para posterior normalização dos dados. Na obtenção das áreas dos sinais utilizou-se a aproximação I $\times\left(\Delta H_{P P}\right)^{2}$ (Poole Jr., 1967), em que I é a intensidade do sinal e $\Delta H_{P P}$ é a largura deste, tomada pico a pico (Figura 1a). Para determinação da concentração relativa dos RLO empregou-se o método do padrão secundário com um cristal de rubi (Singer, 1959) e o padrão "strong pitch" da Bruker com conhecida concentração de spins. Para eliminar a interferência da fração mineral quando da obtenção dos parâmetros espectrais e, portanto, na quantificação dos RLO, foram realizadas análises de RPE dos solos nos quais a matéria orgânica foi calcinada em mufla a $600{ }^{\circ} \mathrm{C}$ por duas horas. Utilizando o software WINEPR Simfonia da Bruker ${ }^{\circledR}$, os espectros foram subtraídos dos espectros das amostras que continham os adubos orgânicos misturados com os solos. Os parâmetros experimentais foram: potência das microondas: $0,2 \mathrm{~mW}$, determinada por experimento de saturação de potência; freqüência de modulação: $100 \mathrm{kHz}$; amplitude de modulação: 0,08 mT; constante de tempo: 2,56 ms e tempo de conversão: $10,24 \mathrm{~ms}$. As análises foram realizadas em duplicata, para
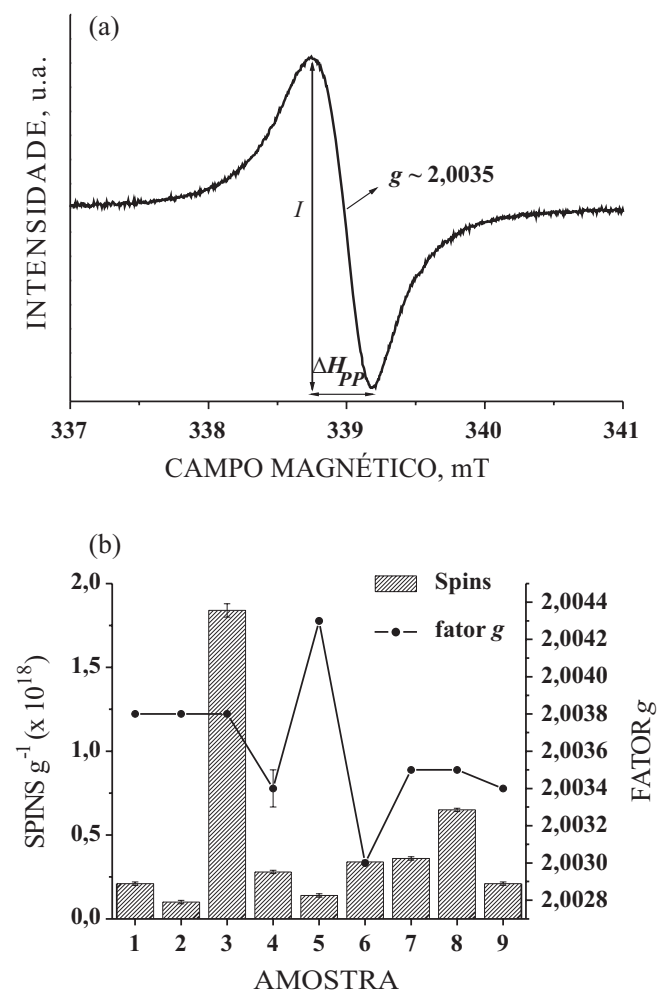

Figura 1. Espectro característico de RPE dos adubos orgânicos (amostra 7), mostrando o sinal do RLO (a) e a concentração de RLO (em spins g $^{-1}$ de amostra) e o valor do fator $g$ das diferentes amostras de adubos orgânicos (b). obtenção do desvio médio com, no mínimo, duas varreduras por determinação. Na obtenção do valor do fator g utilizou-se um capilar contendo $\mathrm{Cr}^{3+}$ em uma estrutura cristalina de $\mathrm{MgO}$ com conhecido valor do fator $\mathrm{g}(1,9797)$. O capilar foi colocado dentro do tubo de quartzo que continha a amostra a qual se desejava determinar o valor do fator $\mathrm{g}$. Fez-se isso para garantir a aquisição dos sinais do $\mathrm{Cr}^{3+}$ e do RLO na mesma freqüência de microondas (Weil et al., 1994).

A análise de componentes principais (PCA) foi aplicada aos valores das concentrações de RLO das nove amostras de adubos orgânicos. Foram analisadas três matrizes: $9 \times 2,8 \times 2$ e $4 \times 2$. Os cálculos foram feitos no software MATLAB $6.1{ }^{\circledR}$.

\section{RESULTADOS E DISCUSSÃo}

$\mathrm{Na}$ figura 1a é apresentado um espectro característico de RPE das amostras de adubos orgânicos (amostra 7), no qual é mostrado o sinal do $\mathrm{RLO}$ com $\Delta H_{P P}$ em torno de $0,4 \mathrm{mT}$ e $\mathrm{g} \approx 2,0035$, estando esses valores próximos dos normalmente encontrados na literatura para ácidos húmicos e fúlvicos (Senesi, 1990). A figura $1 \mathrm{~b}$ apresenta os valores obtidos para a concentração de RLO em spins por massa de amostra (spins $\mathrm{g}^{-1}$ ) e o valor do fator $\mathrm{g}$ dos adubos orgânicos. Esses dados mostram a seletividade da espectroscopia de RPE e o seu potencial para diferenciar as amostras quanto à concentração de RLO, em que se observa grande variação entre os valores obtidos; o maior valor foi para a amostra 3 , que apresentou ordem de grandeza de $10^{18}$ para spins $\mathrm{g}^{-1}$, diferentemente da amostra 2 , cujo valor foi 18,4 vezes inferior.

Para avaliar a significância das variações observadas com relação à concentração de RLO, foi realizada a PCA. A figura 2a, b e c mostra os gráficos PCAs de scores, em que foi possível a distinção entre as amostras (faixas), sendo necessárias apenas duas componentes principais para descrever $100,00 \%$ dos dados. Na figura 2a, 99,97\% da variância total é descrita pela primeira componente principal (PC1) e $0,03 \%$ pela segunda (PC2). Contudo, nessa análise observa-se diferença significativa na amostra 3 , comprometendo a diferenciação das demais amostras. $\mathrm{Na}$ figura $2 \mathrm{~b}$, na qual foi excluída a amostra 3 , $99,73 \%$ da variância total é descrita pela PC1 e $0,27 \%$ pela $\mathrm{PC} 2$. $\mathrm{Na}$ figura $2 \mathrm{~b}$ é possível observar a caracterização de tendências entre as faixas mais claramente. No entanto, a diferenciação entre as faixas 1, 2, 5 e 9 ainda não é evidente, pelo fato de elas estarem agrupadas no primeiro quadrante. $\mathrm{Na}$ figura 2c, onde são analisadas apenas as faixas $1,2,5$ e 9, 99,95\% da variância total é descrita pela PC1 e $0,05 \%$ pela PC2. Nessa última situação observa-se que as faixas ficaram dispersas nos quatro quadrantes, confirmando a possibilidade de diferenciação por meio da concentração de RLO. 

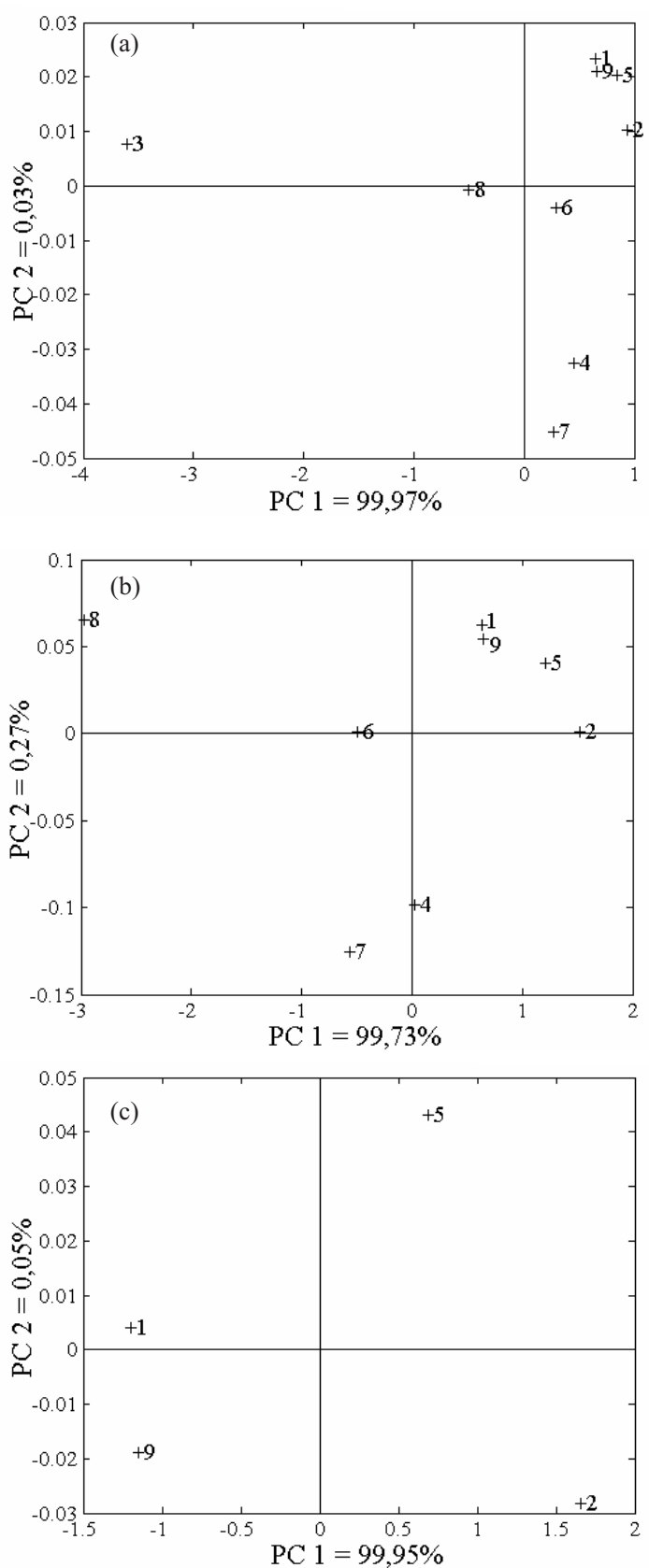

Figura 2. Análise de componentes principais: PC1 x PC2 (scores). Analisando todas as amostras (a); todas as amostras, com exceção da 3 (b); e apenas as amostras 1, 2, 5 e 9 (c).

A determinação da concentração de RLO do solo S1 não foi possível, provavelmente devido à presença de estruturas ferromagnéticas, como o óxido de ferro $\left(\mathrm{Fe}_{2} \mathrm{O}_{3}\right)$, o que é sugerido pela grande quantidade de Fe total (Quadro 2). A presença dessas estruturas impossibilitou a análise quantitativa, por não permitir um ajuste adequado do equipamento de RPE. A adição desse solo à amostra 6 causou deformação na linha de base do sinal do RLO (Figura 3a) e aumento da $\Delta H_{P P}$ (Figura 3b). Essas duas alterações são atribuídas ao surgimento de uma linha larga centrada em $\mathrm{g} \approx 2,2$ (dado não apresentado), gerada pela superposição de linhas decorrentes de interações dipolares entre estruturas ferromagnéticas (Guimarães et al., 2001). Contudo, não se exclui a possibilidade de alargamento espectral associado com o aumento da taxa de relaxação por interação dipolar (Novotny \& Martin-Neto, 2002). Foi observada também variação na concentração de RLO de acordo com a porcentagem de solo adicionado (Figura 3b), ocorrendo acréscimo para a maior porcentagem (20\%) e decréscimo para as menores (1 e $5 \%$ ). Uma hipótese para explicar tal comportamento está relacionada à forma de quantificação dos RLO, na qual se utiliza $\Delta H_{P P}$ elevada ao quadrado; como observado na figura $3 \mathrm{~b}$, a adição do solo causou aumento na $\Delta H_{P P}$ para as maiores porcentagens adicionadas, apesar da subtração espectral para eliminação da interferência da fração mineral. Já o decréscimo para as menores adições deve-se, além do menor aumento de $\Delta H_{P P}$, à diluição da concentração de RLO decorrente da adição do solo que, provavelmente, possui menor concentração desses radicais. Pelo fato
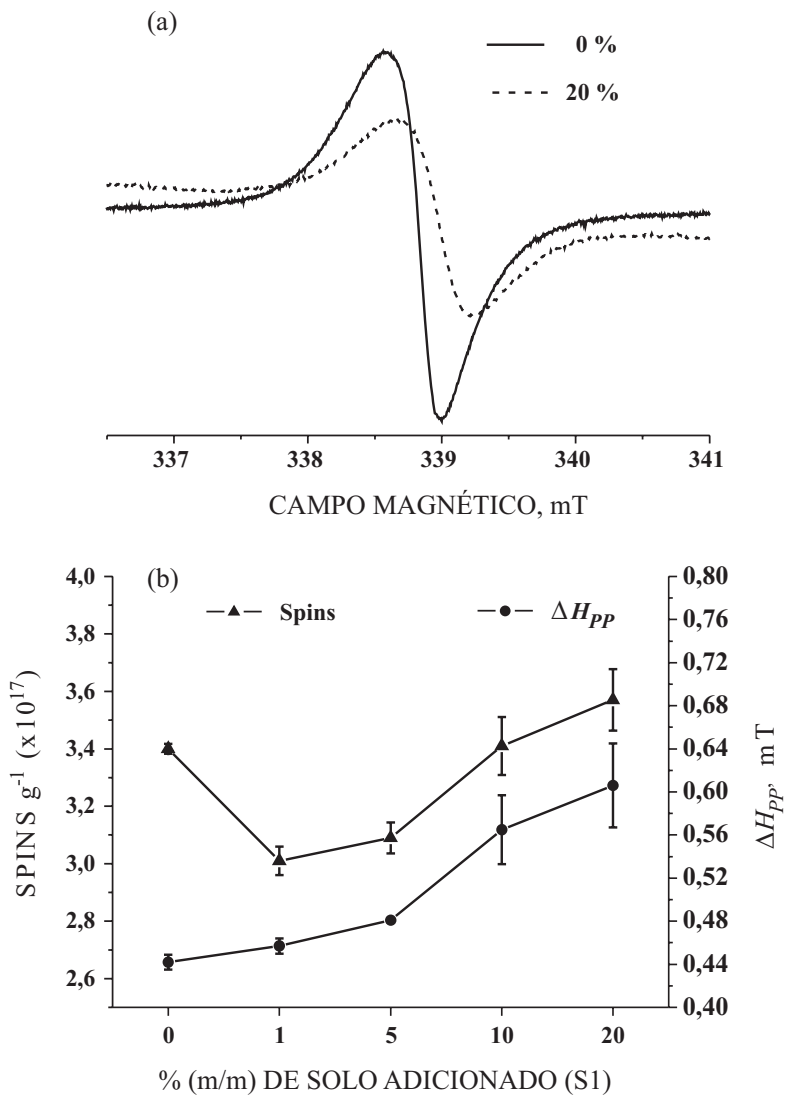

Figura 3. Espectros de RPE da amostra 6 com diferentes porcentagens $(\mathrm{m} / \mathrm{m})$ de solo adicionado (S1) à amostra (a). Largura de linha do sinal $\left(\Delta H_{P P}\right)$ de acordo com a porcentagem de S1 adicionado e concentração de RLO (em spins $\mathrm{g}^{-1}$ de amostra) considerando a porcentagem de solo adicionado (S2) à mesma amostra (b). 
de não terem sido obtidos dados conclusivos, a variação na concentração de RLO não pôde ser usada como um parâmetro para detecção de adulteração quando da adição de solo com estruturas ferromagnéticas.

O valor da concentração de RLO para S2 foi de $4,0 \times 10^{15}$ spins $\mathrm{g}^{-1}$, bastante abaixo dos obtidos para as amostras de adubos orgânicos. A adição desse solo à amostra 6 não causou distorção na linha de base do espectro nem alterações significativas na $\Delta H_{P P}$ (Figura 4a e b, respectivamente). Esse comportamento deve-se à baixa concentração de Fe total (Quadro 2) e, conseqüentemente, de estruturas ferromagnéticas. No entanto, foi possível observar diminuição na concentração de RLO da amostra 6 após a adição desse solo (Figura 4b). A correlação entre a concentração de RLO e a porcentagem de solo adicionado foi linear e inversa, com $r=-0,99(\mathrm{P}<0,0001)$. Isso indica a possibilidade de utilização da concentração de RLO
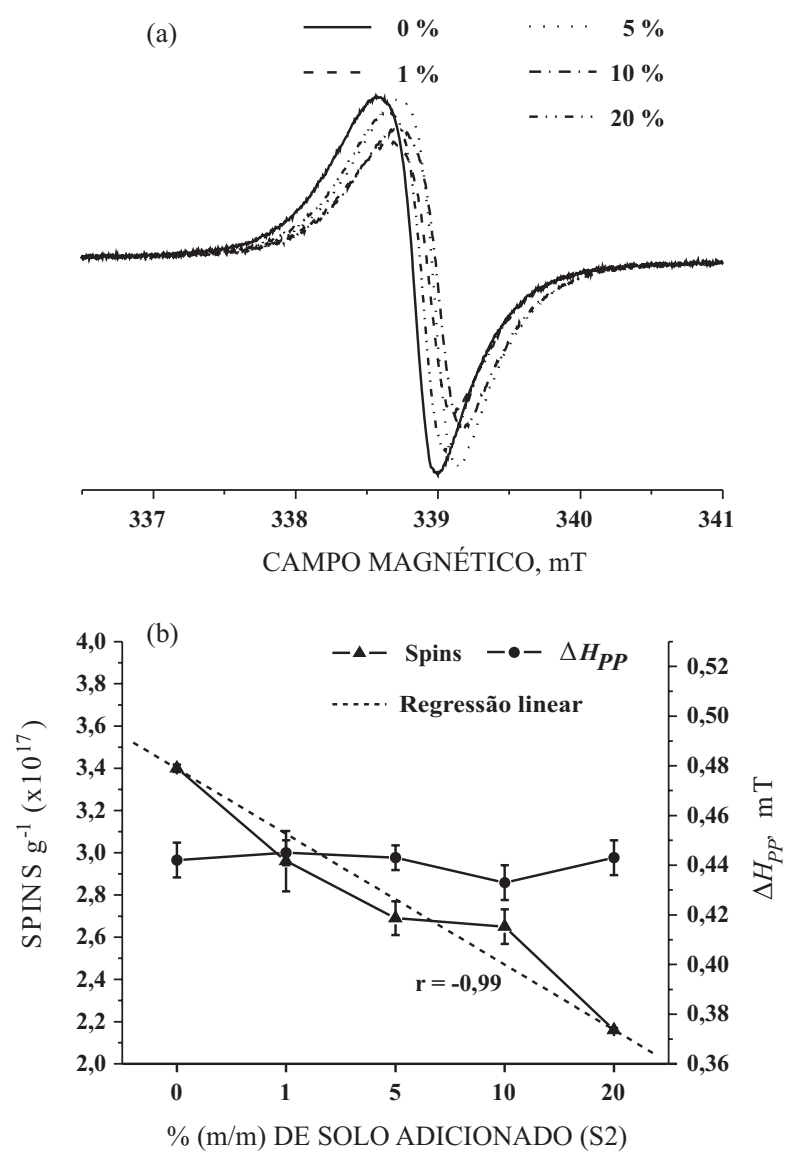

Figura 4. Espectros de RPE da amostra 6 com diferentes porcentagens $(\mathrm{m} / \mathrm{m})$ de solo adicionado (S2) à amostra (a). Concentração de RLO (em spins $\mathrm{g}^{-1}$ de amostra) e largura de linha do sinal $\left(\Delta H_{P P}\right)$ de acordo com a porcentagem de solo adicionado à mesma amostra (b). A regressão linear está associada à concentração de RLO e à porcentagem de solo adicionado. como um parâmetro para detecção de adulteração quando da adição desse tipo de impureza.

Além da diminuição de RLO, o surgimento de novos sinais pode ser um outro indício da presença de impurezas, já que um novo sinal foi detectado quando da adição de S2, o qual foi atribuído ao quartzo $\left(\mathrm{SiO}_{2}\right)$ (Figura 5). O sinal do quartzo apresenta uma linha fina $\left(\Delta H_{P P}=0,1 \mathrm{mT}\right)$ em $\mathrm{g} \approx 2,0000$ (Bersohn \& Baird, 1966), valores esses diferentes do RLO, ou seja, os sinais têm formas e posições diferentes nos espectros de RPE, possibilitando a detecção e diferenciação destes. Analisando os dados (Figura 5), constata-se que foi possível a detecção do quartzo quando adicionados no mínimo $10 \%$ de $\mathrm{S} 2$ e ajustada nos parâmetros experimentais de análise a constante de tempo igual ao tempo de conversão para aumentar a relação sinal/ruído. Além disso, foi utilizado baixo valor de potência das microondas $(0,1 \mathrm{~mW})$, já que o radical livre associado ao quartzo possui pouca interação com o meio onde está localizado, resultando em uma saturação do sinal em baixa potência. Apesar de menos preciso, o surgimento desse novo sinal é um dado que pode ser utilizado para detecção de impurezas contendo quartzo.

O carvão vegetal, quando analisado por RPE, também apresenta um sinal de RLO, sendo a concentração desses radicais normalmente alta. Dessa forma, o carvão vegetal, quando misturado às amostras de adubos orgânicos, pode ocasionar aumento na concentração de RLO dos adubos, gerando valores superestimados. Os resultados comprovaram tal possibilidade, conforme mostrado na figura $6 \mathrm{~b}$, em que é observado aumento na concentração de RLO da amostra 6 após a adição do carvão. Contudo, a presença do carvão pode ser detectada por meio da observação da

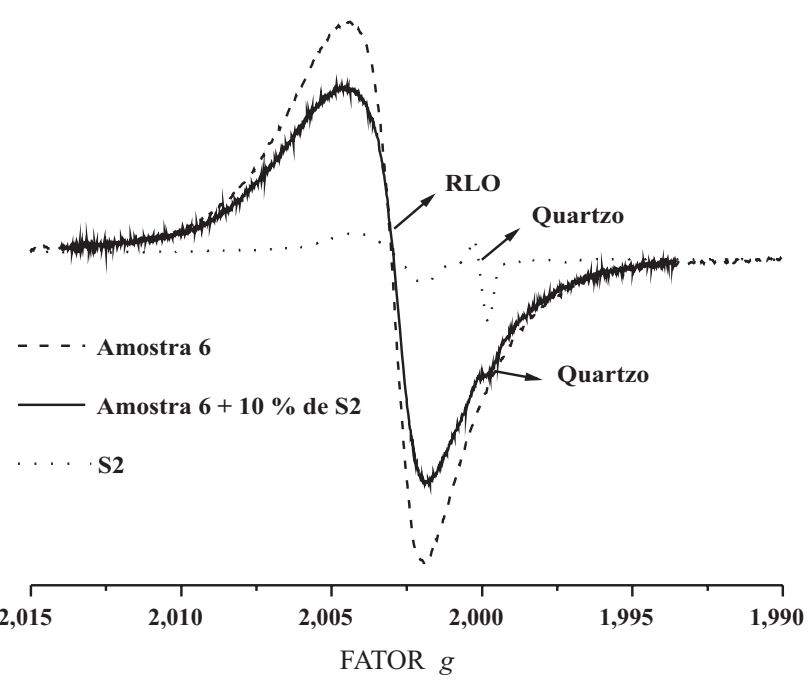

Figura 5. Espectros de RPE da amostra 6, da amostra $6+10 \%(\mathrm{~m} / \mathrm{m})$ de solo $(\mathrm{S2})$ e somente de S2. 
forma de linha do sinal do RLO. Para observar melhor essa forma, foi realizada a integração do espectro de RPE, definida como linha de absorção (Figura 6a). Por essa figura é observado que o sinal do RLO da amostra de adubo orgânico tem alteração espectral após a adição do carvão, passando de uma linha mais lorentziana para uma mais gaussiana, ou seja, tendendo a apresentar mesma forma de linha do sinal do RLO do carvão - essa alteração foi perceptível com no mínimo $10 \%$ de carvão adicionado. Uma possibilidade para a estrutura referente ao sinal do RLO é de semiquinonas possivelmente conjugadas a anéis aromáticos (Schnitzer \& Skinner, 1969; Riffaldi \& Schnitzer, 1972; Senesi \& Steelink, 1989; Senesi, 1990; Stevenson, 1994), nas quais o elétron desemparelhado possui maior probabilidade de estar sobre o átomo de oxigênio. No caso do carvão vegetal, devido à sua estrutura química característica, pode ocorrer maior deslocalização do elétron desemparelhado, fazendo com que o valor do fator $\mathrm{g}$ seja menor (Retcofsky et al., 1968; Schnitzer \& Skinner, 1969). A figura 6b mostra que a adição do carvão vegetal à amostra 7 ocasionou diminuição do valor do fator g da amostra de adubo orgânico, sendo isso perceptível com baixas porcentagens de carvão adicionado. No entanto, a adição do carvão à amostra 6 não alterou o valor do fator $\mathrm{g}$, porque ambas as amostras (amostra 6 e carvão) mostraram valores estatisticamente iguais: $2,0030 \pm 0,0001$ e 2,0029 $\pm 0,0001$, respectivamente. Com isso, a utilização do fator g para detectar a presença de carvão vegetal somente foi possível quando as amostras misturadas apresentaram valores do fator $\mathrm{g}$ significativamente diferentes. Vale ressaltar que, de todas as amostras utilizadas neste trabalho, a 6 foi a única que apresentou valor do fator $\mathrm{g}$ similar ao do carvão vegetal (Figura $1 b$ ).

A utilização direta dos resultados apresentados anteriormente em outras amostras de adubo orgânico não é factível, já que as variações observadas dependeram das características dos solos adicionados. Além disso, parâmetros espectroscópicos, como concentração de RLO, largura e forma de linha do sinal de RPE, e o fator g podem apresentar diferentes variações entre amostras distintas. Apesar disso, os resultados mostraram que é possível detectar a presença de impurezas nas amostras de adubos orgânicos, que a princípio não devem conter partes inorgânicas. No entanto, para análise quantitativa é necessário que se tenha amostra isenta de impurezas, de forma que possibilite uma caracterização espectroscópica para posteriores comparações. Vale ressaltar que, em ambos os solos e em todas as adições realizadas, não foi possível detectar diferenças visuais (a olho nu) entre a amostra original e aquelas às quais foram adicionados solos ou carvão vegetal. Ademais, as alterações espectroscópicas supracitadas foram similares entre as duas amostras (6 e 7), com exceção do fator $g$, mostrando a potencialidade da espectroscopia de RPE como uma ferramenta para qualificação de adubos orgânicos. (a)

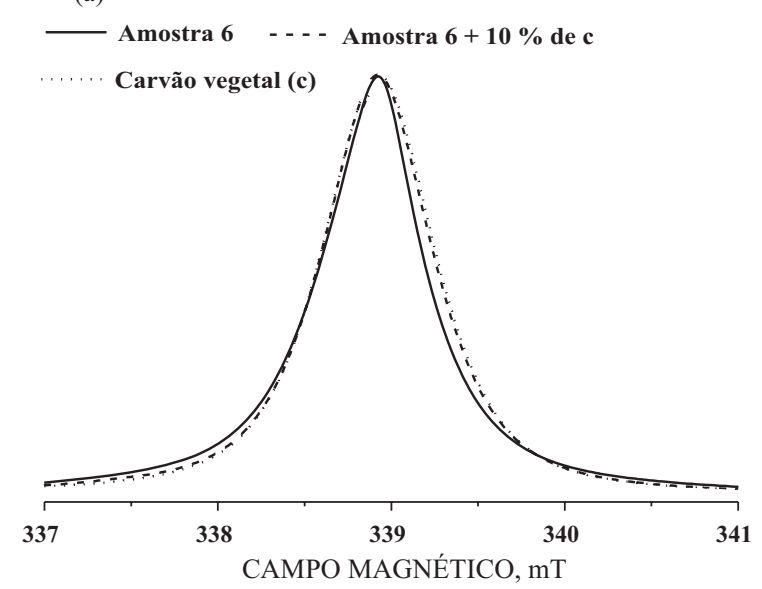

(b)

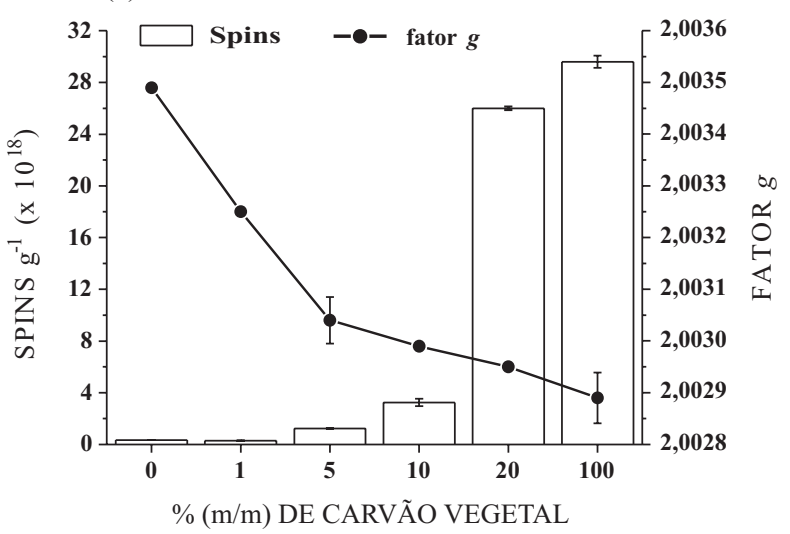

Figura 6. Linhas de absorção normalizadas da amostra 6 , da amostra $6+10 \%(\mathrm{~m} / \mathrm{m})$ de carvão vegetal e somente do carvão vegetal (a). Concentração de RLO (em spins g $^{-1}$ de amostra) da amostra 6 e variação do fator $g$ da amostra 7 de acordo com a porcentagem de carvão vegetal adicionado (b).

\section{CONCLUSÕES}

1. Com relação à necessidade de parâmetros que permitam a padronização e qualificação de adubos orgânicos, observou-se que a concentração de RLO tem potencial para diferenciar as amostras de adubos orgânicos. A significância da variação da concentração de RLO entre as amostras foi comprovada com a aplicação de PCA.

2. As alterações espectrais com relação ao sinal do RLO - como deformação da linha de base, alteração na largura de linha $\left(\Delta H_{P P}\right)$, variação na concentração de RLO, surgimento de novos sinais, alteração na forma de linha de absorção e do valor do fator $\mathrm{g}$ - podem ser utilizadas para detectar adulterações provenientes da adição de solos ou carvão vegetal às amostras de adubos orgânicos.

3. A largura de linha e a distorção na linha de base do sinal de RPE foram os parâmetros mais adequados para detectar a presença de impurezas com 
estruturas paramagnéticas. A concentração de RLO e o surgimento de novos sinais de RPE foram os mais adequados para impurezas contendo quartzo e isentas de estruturas paramagnéticas. Quando a impureza adicionada foi o carvão vegetal, os parâmetros mais adequados foram concentração de RLO e fator $\mathrm{g}$.

\section{AGRADECIMENTOS}

À Embrapa Instrumentação Agropecuária, pela infra-estrutura cedida, e à Embrapa Pecuária Sudeste, pelas análises dos solos.

\section{LITERATURA CITADA}

ATIYEH, R.M.; EDWARDS, C.A.; SUBLER, S. \& METZGER, J.D. Pig manure vermicompost as a component of a horticultural bedding plant medium: Effects on physicochemical properties and plant growth. Biores. Technol., 78:11-20, 2001.

BAYER, C.; MIELNICZUK, J.; MARTIN-NETO, L. \& ERNANI, P.R. Stocks and humification degree of organic matter fractions as affected by no-tillage on a subtropical soil. Plant Soil, 238:133-140, 2002.

BERSOHN, M. \& BAIRD, J.C. An introduction to electron paramagnetic resonance. New York, W.A. Benjamin, 1966. $272 \mathrm{p}$.

CERETTA, C.A.; BAYER, C.; DICK, D.P.; MARTIN-NETO, L. \& COLNAGO, L.A. Métodos espectroscópicos. In: SANTOS, G.A. \& CAMARGO, F.A.O., eds. Fundamentos da matéria orgânica do solo: Ecossistemas tropicais e subtropicais. Porto Alegre, Gênesis, 1999. 491p.

DICK, D.P.; MANGRICH, A.S.; MENEZES, S.M.C. \& PEREIRA, B.F. Chemical and spectroscopical characterization of humic acids from two south Brazilian coals of different ranks. J. Braz. Chem. Soc., 13:177-182, 2002.

GRIGATTI, M.; CIAVATTA, C. \& GESSA, C. Evolution of organic matter from sewage sludge and garden trimming during composting. Biores. Technol., 91:163-169, 2004.

GUIMARÃES, E.; MANGRICH, A.S.; MACHADO, V.G.; TRAGHETTA, D.G. \& LOBO, M.A. Criterious preparation and characterization of earthworm-composts in view of animal waste recycling. Part II. A synergistic utilization of EPR and H-1 NMR spectroscopies on the characterization of humic acids from vermicomposts. J. Braz. Chem. Soc., 12:734-741, 2001.

JERZYKIEWICZ, M.; DROZD, J. \& JEZIERSKI, A. Organic radicals and paramagnetic metal complexes in municipal solid waste composts. An EPR and chemical study. Chemosphere, 39:253-268, 1999.

MARTIN-NETO, L.; ANDRIULLO, A.E. \& TRAGHETTA, D.G. Effects of cultivation on ESR spectra of organic matter from soil size fractions of a mollisol. Soil Sci., 157:365372,1994
MARTIN-NETO, L.; NASCIMENTO, O.R.; TALAMONI, J. \& POPPI, N.R. EPR of micronutrients-humic substance complexes extracted from Braz. Soil. Soil Sci., 151:369$376,1991$.

MARTIN-NETO, L.; ROSELL, R. \& SPOSITO, G. Correlation of spectroscopic indicators of humification with mean annual rainfall along a temperate grassland climosequence. Geoderma, 81:305-311, 1998.

MIYASAKA, S.; OHKAWARA, T.; NAGAI, K.; YAZAKI, H. \& SAKITA, M.N. Técnicas de produção e uso do fino de carvão e licor pirolenhoso. In: ENCONTRO DE PROCESSOS DE PROTEÇÃO DE PLANTAS: Controle ecológico de pragas e doenças, 1., Botucatu, 2001. Resumo. Botucatu, 2001. p.161-176.

NOVOTNY, E. H. \& MARTIN-NETO, L. Effects of humidity and metal ions on the free radicals analysis of peat humus. Geoderma, 106:305-317, 2002.

OLK, D.C.; BRUNETTI, G. \& SENESI, N. Decrease in humification of organic matter with intensified lowland rice cropping: A wet chemical and spectroscopic investigation. Soil Sci. Soc. Am. J., 64:1337-1347, 2000.

PAJACZKOWSKA, J.; SULKOWSKA, A.; SULKOWSKI, W.W. \& JEDRZEJCZYK, M. Spectroscopy study of the humification process during sewage sludge treatment. J. Molec. Struc., 651-653:141-149, 2003.

PEREZ, M.G.; MARTIN-NETO, L.; SAAB, S.C.; NOVOTNY, E.H.; MILORI, D.M.B.P.; BAGNATO, V.S.; COLNAGO, L.A.; MELO, W.J. \& KNICKER, H. Characterization of humic acids from a Brazilian Oxisol under different tillage systems by EPR, C-13 NMR, FTIR and fluorescence spectroscopy. Geoderma, 118:181-190, 2004.

PLAZA, C.; SENESI, N.; BRUNETTI, G. \& MONDELLI, D. Cocomposting of sludge from olive oil mill wastewater mixed with tree cuttings. Compost Sci. Utiliz., 13:217226,2005

PLAZA, C.; SENESI, N.; POLO, A.; BRUNETTI, G.; GARCIAGIL, J.C. \& D'ORAZIO, V. Soil fulvic acid properties as a mean to assess the use of pig slurry amendment. Soil Till. Res., 74:179-190, 2003.

POOLE Jr., C.P. Electron spin resonance: A comprehensive treatise on experimental techniques. New York, WileyInterscience, 1967. 921p.

RETCOFSKY, H.L.; STARK, J.M. \& FRIEDEL, R.A. Electron spin resonance in American coals. Anal. Chem., 40:16991794,1968 .

RIFFALDI, R. \& SCHNITZER, M. Electron spin resonance spectrometry of humic substances. Soil Sci. Soc. Am., 36:301-305, 1972.

ROVIRA, P.A.S.; BRUNETTI G.; POLO, A. \& SENESI, N. Comparative chemical and spectroscopic characterization of humic acids from sewage sludges and sludge-amended soils. Soil Sci., 167:235-245, 2002.

SAAB, C.S. \& MARTIN-NETO, L. Use of the EPR technique to determine thermal stability of some humified organic substances found in soil organic-mineral fractions. Química Nova, 26:497-498, 2003. 
SCHNITZER, M. \& LEVESQUE, M. Electron spin resonance as a guide to the degree of humification of peats. Soil Sci., $127: 140-145,1979$

SCHNITZER, M. \& SKINNER, S.I.M. Free radicals in soil humic compounds. Soil Sci., 108:383-390, 1969.

SENESI, N. Application of electron spin resonance (ESR) spectroscopy in soil chemistry. Adv. Soil. Sci., 14:77-130, 1990.

SENESI, N. \& STEELINK, C. Application of ESR spectroscopy to the study of humic substances. In: HAYES, M.H.B.; MacCARTHY, P.; MALCOLM, R.L. \& SWIFT, R.S. Humic substances II. In search of structure. New York, J. Wiley, 1989. p.373-407.

SINGER, L.S. Synthetic ruby as a secondary standard for the measurement of intensities in electron paramagnetic resonance. J. Appl. Phys., 30:1463-1464, 1959.

STEVENSON, F.J. Humus chemistry: Genesis, composition, reactions. New York, J. Willey, 1994. 496p.
TOMATI, U.; BELARDINELLI, M.; ANDREU, M.; GALLI, E.; CAPITANI, D.; PROIETTI, N. \& De SIMONE, C. Evaluation of commercial compost quality. Waste Manag. Res., 20:389-397, 2002.

TSUZUKI, E.; MORIMITSU, T. \& MATSUI, T. Effect of chemical compounds in pyroligneous acid on root growth in rice plant. Japan J. Crop. Sci., 66:15-16, 2000.

WEIL, J. A.; BOLTON, J. R. \& WERTZ, J.E. Electron paramagnetic resonance: Elementary theory and practical applications. New York, J. Willey, 1994. 568p.

ZANETTI, M.; CAZETTA, J.O.; MATOS JUNIOR, D. \& CARVALHO, S.A. Uso de subprodutos de carvão vegetal na formação do porta-enxerto limoeiro 'cravo' em ambiente protegido. R. Bras. Frutic., 25:508-512, 2003.

ZECH, W.; SENESI, N.; GUGGENBERG, G.; KAISER, K.; LEHMANN, J.; MIANO, T.M.; MILTNER, A. \& SCHROTH, G. Factors controlling humification and mineralization of soil organic matter in the tropics. Geoderma, 79:117-161, 1997. 